\title{
CORRELAÇÃO ENTRE CARACTERÍSTICAS DE LATOSSOLOS E PARÂMETROS DE EQUAÇÕES DE ADSORÇÃO DE COBRE E ZINCO(1)
}

\author{
C. W. A. NASCIMENTO ${ }^{(2)} \&$ R. L. F. FONTE $S^{(3)}$
}

\begin{abstract}
RESUMO
A adsorção de $\mathrm{Cu}$ e $\mathrm{Zn}$ é um dos principais fenômenos responsáveis pela disponibilidade desses elementos para as plantas. Portanto, estudos sobre a adsorção desses elementos em solos podem ser ferramentas úteis para compreensão da relação entre características dos solos e retenção desses micronutrientes. Nesse sentido, o presente trabalho objetivou caracterizar a adsorção de Cu e Zn em amostras de seis Latossolos de Minas Gerais e as influências de características dos solos sobre os parâmetros de adsorção obtidos pelas equações de Langmuir e de Freundlich. Para tanto, foram utilizadas soluções de cloreto de cobre e cloreto de zinco nas concentrações $0,10,20,40,60$, $80,100,120$ e $140 \mathrm{mg} \mathrm{L}^{-1}$, ajustadas a pH 5,5. As equações de Langmuir e F reundlich foram eficientes na determinação dos parâmetros de adsorção de Zn e Cu, sendo os teores de argila e de matéria orgânica, respectivamente, as características mais bem relacionadas com a capacidade de adsorção desses elementos pelos solos. Os solos estudados apresentaram mai or energia de li gação dos sítios de troca e maior capacidade de adsorção máxima para Cu relativamente ao $\mathrm{Zn}$. O P remanescente não se correlacionou com os parâmetros de adsorção de $\mathrm{Zn}$, em virtude, provavelmente, da influência da matéria orgânica em sua determinação. Este fato pode limitar sua eficiência na previsão da adsorção deste elemento em solos com alto teor de C orgânico.
\end{abstract}

Termos de indexação: isotermas de adsorção, micronutrientes, Langmuir, Freundlich.

(1) Recebido para publicação em fevereiro de 2003 e aprovado em outubro de 2004.

(2) Professor do Departamento de Agronomia da Universidade Federal Rural de Pernambuco - UFRPE. Rua D. Manuel de Medeiros $\mathrm{s} / \mathrm{n}$, Dois I rmãos, CEP 52171-900 Recife (PE). E-mail: cwanascimento@yahoo.com

(2) Professor do Departamento de Solos da Universidade Federal de Viçosa - UFV. Av. PH Rolfs s/n, CEP $36570-000$ Viçosa (MG). E-mail: renildes@solos.ufv.br 


\title{
SUMMARY: CORRELATION BETWEEN CHARACTERISTICS OF LATOSOLS FROM MINAS GERAIS STATE, BRAZIL, AND PARAMETERS OF ADSORPTION EQUATIONS OF COPPER AND ZINC
}

\begin{abstract}
Copper and zinc adsorption is one of the main phenomena controlling theavailability of these metals to plants. As a consequence, studies on the adsorption of such elements in soils may be useful tools to understand the relationship between soil characteristics and retention of thesemi cronutrients. Thepresent study exami ned theadsorption of copper and zinc in six Latosols (Oxisols) with a widerange of chemical properties. Solutions containing different $\mathrm{Cu}$ and $\mathrm{Zn}$ concentrations $\left(0,10,20,40,60,80,100,120\right.$, and $\left.140 \mathrm{mg} \mathrm{L}^{-1}\right)$ were equilibrated with soil samples to determine whether $\mathrm{Cu}$ and $\mathrm{Zn}$ adsorption conformed to the Langmuir and Freundlich isotherms, and to relate the isotherm coefficients to soil properties. The Langmuir and Freundlich wereadequatefor providing $\mathrm{Cu}$ and $\mathrm{Zn}$ adsorption coefficients. Theclay and organic matter contents, respectively, werethesoil characteristics that best correlated to $\mathrm{Cu}$ and $\mathrm{Zn}$ adsorption to soils. The studied soils presented both higher adsorption energy and higher adsorption capacity for $\mathrm{Cu}$ than they did for $\mathrm{Zn}$. The remaining $\mathrm{P}$ was not correlated to $\mathrm{Zn}$ adsorption coefficients probably due, to the influence of organic matter on its solution equilibrium $P$ determination. This fact may limit the remaining $\mathrm{P}$ ability to predict $\mathrm{Zn}$ adsorption to soils with high organic matter content.
\end{abstract}

Index terms: adsorption isotherms, micronutrients, Latosols.

\section{INTRODUÇÃO}

Sintomas de deficiência de Cu e de Zn têm sido relatados em diversas culturas, em várias regiões do Brasil (Machado \& Pavan, 1987; Lopes \& Abreu, 2000). Em Latossolos, essas deficiências podem ser atribuídas à pequena reserva natural desses micronutrientes e às suas baixas disponibilidades, os quais se encontram, predominantemente, nas formas oxídicas e residuais, não-disponíveis às plantas (Nascimento, 2001). A adsorção é, talvez, o principal processo a ser considerado para o entendimento da disponibilidade de Cu e Zn, visto que dá indicativos da solubilidade e mobilidade desses el ementos e a conseqüente disponibilidade para as plantas. De acordo com Harter (1991), as reações de adsorção-dessorção tendem a ser mais rápidas que os processos de preci pitação-dissolução, de modo que a adsorção na interface sól ido-solução pode ser o fator dominante para regular a concentração de micronutrientes em solução.

Micronutrientes podem ser adsorvidos por atração el etrostática na dupla camada difusa (adsorção não-específica) ou pela complexação de superfície (adsorção específica), processo no qual os solutos se ligam à matriz do solo para formar complexos de esfera interna, onde os cátions são retidos fortemente por ligações covalentes (Selim, 1992), com diminuição acentuada das suas labilidades.

I sotermas de adsorção têm sido amplamente utilizadas para estudos dos fenômenos de adsorção (Msaky \& Calvet, 1990; Yuan \& Lavkulich, 1997), sendo as equações de L angmuir e de Freundlich as mais utilizadas. Essas equações, embora não esclareçam sobre os mecanismos químicos envolvidos na adsorção, são facilmente aplicáveis para adsorção de quaisquer íons pelo solo e têm a vantagem de fornecer parâmetros que possam ser relacionados com propriedades dos sol os. A adsorção de $\mathrm{Cu}$ e de $\mathrm{Zn}$ varia entre solos e pode ser influenciada por diversas propriedades, tais como: $\mathrm{pH}$, teor de matéria orgânica, composição mineralógica e textura (Shuman, 1975; McBride \& Blasiak, 1979; Martínez \& Motto, 2000) ou pela competição entre íons pelas superfícies adsorventes (Bibak, 1997; Mesquita, 1998; Echeverría et al., 1998).

As isotermas de Langmuir e Freundlich têm-se mostrado adequadas para estudos de adsorção de Cu e Zn em solos do Brasil (Pombo \& Klamt, 1986; Machado \& Pavan, 1987; Cunha et al., 1994; Silveira \& Alleoni, 2003). Nesses estudos, em geral, características dos solos, tais como: CTC (Pombo \& Klamt, 1986; Silveira \& Alleoni, 2003), matéria orgânica e óxidos de Fe (Silveira et al., 2002) têm apresentado boas correl ações com os parâmetros das equações de adsorção. Machado \& Pavan (1987), entretanto, não observaram relação entre matéria orgânica ou teor de argila com parâmetros de adsorção de Zn. Para Cu, Silveira et al. (2002) observaram aumento na adsorção após remoção dos óxidos de $\mathrm{Fe}$ e redução quando da remoção de matéria orgânica.

Visto que as deficiências de Cu e de Zn situam-se entre as mais importantes em solos brasileiros, os mecanismos de adsorção desses micronutrientes para essas condições são importantes para previsão da disponibilidade. Com este objetivo, foi realizado um experimento em laboratório, para caracterizar a adsorção de $\mathrm{Cu}$ e de $\mathrm{Zn}$ em Latossolos de Minas Gerais e as influências de características dos solos 
sobre os parâmetros de adsorção obtidos pelas equações de Langmuir e de Freundlich.

\section{MATERIAL E MÉTODOS}

Para os ensaios, foram selecionados seis Latossolos de diferentes municípios do estado de Minas Gerais, com teores diferentes de argila e de matéria orgânica, os quais foram classificados como Latossolo Vermelho - LV1 (Sete Lagoas), Latossolo Vermelho - LV2 (São Sebastião do Paraíso), Latossolo Vermelho - LV3 (São Sebastião do Paraíso), Latossolo Vermel ho-Amarelo - LVA1 (J oão Pinheiro), Latossolo Vermel ho-Amarelo - LVA2 (Viçosa) e Latossolo Vermelho-Amarelo húmico LVA3 (Viçosa).

Amostras da camada arável dos solos $(0-20 \mathrm{~cm})$ foram destorroadas, secas ao ar e passadas em peneiras de $2 \mathrm{~mm}$ de diâmetro de malha. Em seguida, as amostras foram caraterizadas física e quimicamente (Quadro 1), enquanto a composição mineralógica (Quadro 2) foi estimada a partir dos teores de óxidos totais pel o ataque sulfúrico da terra fina seca ao ar (Resende et al., 1987). Houve predominância de caulinita nos solos LV1, LVA1, LVA2 e LVA3 e deóxidos de Fe (goethita e hematita) e gibbsita nos sol os LV2 e LV3; baixos val ores de Ki e $\mathrm{Kr}$ indicam o el evado grau de intemperismo das amostras dos solos.

As curvas de adsorção de $\mathrm{Cu}$ e de $\mathrm{Zn}$ nos solos foram determinadas pela adição de $25 \mathrm{~mL}$ de uma solução que continha as seguintes concentrações: $0,10,20,40,60,80,100,120$ e $140 \mathrm{mg} \mathrm{L}^{-1}$ do el emento, na forma de $\mathrm{CuCl}_{2} \cdot 2 \mathrm{H}_{2} \mathrm{O}$ ou de $\mathrm{ZnCl}_{2}$, em $0,01 \mathrm{~mol} \mathrm{~L}^{-1}$ de $\mathrm{CaCl}_{2} \cdot 2 \mathrm{H}_{2} \mathrm{O}$, a $1,0 \mathrm{~g}$ de solo (TFSA) em tubos de centrífuga de $50 \mathrm{~mL}$, em três repetições. As soluções tiveram seus valores de $\mathrm{pH}$ corrigidos para 5,5 imediatamente antes do início do ensaio. As suspensões sol o-solução foram agitadas por $16 \mathrm{~h}$ a 120 rpm e, em seguida, centrifugadas, filtradas e analisadas, para Cu eZn, por espectrofotometria de absorção atômica. As concentrações de Cu e de Zn adsorvidas foram consideradas a diferença entre aquelas inicialmenteadi cionadas eas remanescentes após o período de equilíbrio.

Quadro 1. Caracterização química e física das amostras de solos provenientes de Sete Lagoas (LV1), São Sebastião do Paraíso (LV2 e LV3), J oão Pinheiro (LVA1) e Viçosa (LVA2 e LVA3)

\begin{tabular}{|c|c|c|c|c|c|c|}
\hline Característica & LV1 & LV2 & LV3 & LVA1 & LVA2 & LVA3 \\
\hline $\mathrm{pH} \mathrm{H} \mathrm{H}_{2} \mathrm{O}(1: 2,5)$ & 4,6 & 4,4 & 4,7 & 4,0 & 4,1 & 4,2 \\
\hline$P\left(\mathrm{mg} \mathrm{dm}^{-3}\right)^{(1)}$ & 4,0 & 0,0 & 0,0 & 1,3 & 0,0 & 1,9 \\
\hline $\mathrm{K}\left(\mathrm{mg} \mathrm{dm}^{-3}\right)^{(1)}$ & 20 & 59 & 16 & 21 & 15 & 15 \\
\hline $\mathrm{Ca}^{2+}\left(\mathrm{cmol}_{\mathrm{c}} \mathrm{dm}^{-3}\right)^{(2)}$ & 1,24 & 0,55 & 0,72 & 0,08 & 0,02 & 0,46 \\
\hline $\mathrm{Mg}^{2+}\left(\mathrm{cmol}_{\mathrm{c}} \mathrm{dm}^{-3}\right)^{(2)}$ & 0,20 & 0,11 & 0,23 & 0,04 & 0,02 & 0,13 \\
\hline $\mathrm{Al}^{3+}\left(\mathrm{cmol}_{\mathrm{c}} \mathrm{dm}^{-3}\right)^{(2)}$ & 0,50 & 0,10 & 0,20 & 0,90 & 1,20 & 1,90 \\
\hline $\mathrm{H}+\mathrm{Al}\left(\mathrm{cmol}_{\mathrm{c}} \mathrm{dm}^{-3}\right)$ & 8,3 & 11,9 & 6,6 & 6,6 & 8,9 & 7,6 \\
\hline $\mathrm{Zn}\left(\mathrm{mg} \mathrm{dm}^{-3}\right)^{(1)}$ & 1,21 & 0,46 & 0,37 & 0,40 & 0,18 & 1,14 \\
\hline $\mathrm{Cu}\left(\mathrm{mg} \mathrm{dm} \mathrm{dm}^{-3}\right)^{(1)}$ & 0,77 & 6,57 & 8,73 & 0,40 & 0,12 & 0,22 \\
\hline Zn total $\left(\mathrm{mg} \mathrm{dm}^{-3}\right)$ & 58,25 & 104,43 & 115,03 & 12,86 & 11,77 & 20,12 \\
\hline Cu total $\left(\mathrm{mg} \mathrm{dm}^{-3}\right)^{(4)}$ & 43,35 & 102,83 & 125,38 & 16,47 & 21,75 & 11,70 \\
\hline $\mathrm{CTC}_{\text {efetiva }}\left(\mathrm{cmol}_{\mathrm{c}} \mathrm{dm}^{-3}\right)$ & 2,45 & 0,91 & 1,19 & 1,07 & 1,28 & 2,53 \\
\hline CTC total $\left(\mathrm{cmol}_{\mathrm{c}} \mathrm{dm}^{-3}\right)$ & 10,25 & 12,71 & 7,59 & 6,77 & 8,98 & 8,23 \\
\hline$V(\%)$ & 14,5 & 6,4 & 13,0 & 2,5 & 0,87 & 7,7 \\
\hline $\mathrm{m}(\%)$ & 20,4 & 11,0 & 16,8 & 84,1 & 93,81 & 75,1 \\
\hline C.O. $\left(\mathrm{g} \mathrm{kg}^{-1}\right)$ & 28,7 & 13,92 & 17,0 & 8,7 & 18,6 & 112,5 \\
\hline P-rem (mg L-1) (6) & 7,48 & 3,61 & 6,54 & 28,65 & 2,76 & 1,34 \\
\hline $\mathrm{SiO}_{2}\left(\text { dag kg }^{-1}\right)^{(7)}$ & 18,3 & 4,8 & 7,4 & 12,8 & 19,8 & 10,2 \\
\hline $\mathrm{Al}_{2} \mathrm{O}_{3}\left(\right.$ dag kg $\left.^{-1}\right)$ & 31,80 & 34,11 & 32,10 & 16,87 & 28,85 & 19,05 \\
\hline $\mathrm{Fe}_{2} \mathrm{O}_{3}\left(\text { dag kg }^{-1}\right)^{(7)}$ & 12,42 & 33,84 & 30,41 & 4,60 & 12,66 & 5,37 \\
\hline $\mathrm{TiO}_{2}(\text { dag kg-1 })^{(7)}$ & 0,71 & 6,75 & 6,15 & 0,51 & 1,93 & 0,64 \\
\hline $\mathrm{MnO}($ dag kg-1) (7) & 0,03 & 0,15 & 0,15 & 0,01 & 0,01 & 0,01 \\
\hline $\mathrm{P}_{2} \mathrm{O}_{5}\left(\text { dag kg }^{-1}\right)^{(7)}$ & 0,21 & 0,24 & 0,22 & 0,03 & 0,06 & 0,11 \\
\hline Areia grossa $\left(\mathrm{gkg}^{-1}\right)$ & 110 & 70 & 90 & 400 & 150 & 460 \\
\hline Areia fina $\left(\mathrm{g} \mathrm{kg}^{-1}\right)$ & 40 & 230 & 300 & 230 & 100 & 140 \\
\hline Silte $\left(\mathrm{g} \mathrm{kg}^{-1}\right)$ & 140 & 240 & 190 & 50 & 70 & 160 \\
\hline Argila $\left(\mathrm{g} \mathrm{kg}^{-1}\right)$ & 710 & 460 & 420 & 320 & 680 & 240 \\
\hline
\end{tabular}

(1) Mehlich-1 (De Filippo \& Ribeiro, 1997). (2) KCl 1 mol L-1 (De Filippo \& Ribeiro, 1997). (3) CaOAc 0,5 mol L ${ }^{-1}$ (De Filippo \& Ribeiro, 1997). ${ }^{(4)}$ Ataque triácido (Ure, 1990). ${ }^{(5)}$ Walkley-Black (De Filippo \& Ribeiro, 1997). ${ }^{\left({ }^{6}\right)}$ Alvarez V. et al. (2000). ${ }^{(7)}$ Ataque sulfúrico (Vettori, 1969). ${ }^{(8)}$ Embrapa (1997). 


\section{Quadro 2. Mineralogia da fração argila, estimada a partir dos teores de óxidos pelo ataque sulfúrico, e valores de $\mathrm{Ki}$ e $\mathrm{Kr}$ das amostras dos solos}

\begin{tabular}{|c|c|c|c|c|c|c|}
\hline Solo & Caulinita & Hematita & Goethita & Gibbsita & $\mathbf{K i}$ & $\mathbf{K r}$ \\
\hline & \multicolumn{4}{|c|}{ - \% (1) } & & \\
\hline LV1 & 39,40 & 7,05 & 9,32 & 22,60 & 0,98 & 0,78 \\
\hline LV2 & 10,37 & 16,55 & 21,88 & 40,67 & 0,24 & 0,15 \\
\hline LV3 & 15,85 & 23,75 & 31,41 & 32,01 & 0,39 & 0,24 \\
\hline LVA1 & 27,42 & 5,82 & 7,70 & 7,36 & 1,29 & 1,09 \\
\hline LVA2 & 42,69 & 10,92 & 14,44 & 14,88 & 1,17 & 0,91 \\
\hline LVA3 & 22,04 & 5,23 & 6,92 & 14,16 & 0,91 & 0,77 \\
\hline
\end{tabular}

(1) De acordo com Resende et al. (1987).

A equação de $L$ angmuir, $C / x / m=1 / k b+C / b$, foi ajustada aos dados experimentais de adsorção de Cu e de Zn. Nessa equação, $C=$ concentração de Zn ou Cu na solução de equilíbrio em $\mathrm{mg} \mathrm{L}^{-1}, \mathrm{x} / \mathrm{m}=$ quantidade de Zn ou Cu adsorvida em $\mathrm{mg} \mathrm{g}^{-1}$ de solo, $\mathrm{b}=$ adsorção máxima $\left(\mathrm{mg} \mathrm{g}^{-1}\right)$ e $\mathrm{k}=$ constante relacionada com a energia de ligação $\left(L \mathrm{mg}^{-1}\right)$ de $\mathrm{Zn}$ ou Cu no solo. Após linearização da equação (C/x/m em função de $C)$, a declividade (1/b) e o intercepto $(1 / k b)$ foram usados para calcular a capacidade de adsorção máxima (b) ea constante relacionada com a energia de ligação $(\mathrm{k})$. A equação de Freundlich $\left(\mathrm{x} / \mathrm{m}=\mathrm{k} \mathrm{C}^{\mathrm{l} / \mathrm{n}}\right)$, na qual $\mathrm{k}$ éo coeficiente de equilíbrio e o parâmetro $1 / n$ é admensional, foi linearizada com $\log x / m$ em função de $\log C$, produzindo uma reta com decl ividade $1 / n$ e intercepto log $k$, os quais foram utilizados para calcular os valores $n$ ek.

A significância estatística dos coeficientes de correlação para $\mathrm{C} / \mathrm{x} / \mathrm{m}$ em função de C (Langmuir) e $\log x /$ m em função de $\log C$ (Freundlich) foi o critério pelo qual os dados das isotermas foram testados.

\section{RESULTADOS E DISCUSSÃO}

Os ajustes dos modelos de isotermas aos dados experimentais foram bons e em apenas um caso o coeficiente de determinação foi menor que 0,90 (Quadro 3). As equaçõs de Langmuir e Freundlich têm si do usadas por diversos autores (Shuman, 1975; Petruzzeli et al., 1985; Machado \& Pavan, 1987; Yuan \& Lavkulich, 1997) com coeficientes de determinaçãotão altos quanto os obtidos no presente trabalho, o que indica que esses model os são bem ajustados a diversas condições de solo e concentrações de metais.

As relações entre os teores de Cu na solução de equilíbrio e os adsorvidos nos solos (Figura 1 ) demonstram que os sol os LVA3 e LV 1 apresentam o tipo " $\mathrm{H}$ " de isoterma, conforme classificação de Giles et al. (1974), citados por Harter (1991), o que indica alta afinidade deadsorção, enquanto os demais sol os apresentam isotermas dotipo " $L$ ", com menor energia de adsorção. Essas características das isotermas dependem, provavel mente, das propriedades dos solos, parecendo estar relacionadas com os teores de matéria orgânica, em virtude da elevada afinidade desta com o Cu (McLaren \& Crawford, 1973). Tais resultados podem, também, ser corroborados pelas mais el evadas capacidades máximas de adsor ção de Cu, obtidas após ajuste da equação de Langmuir, queforam de 2,08 e 1,70 $\mathrm{mg} \mathrm{g}^{-1}$ (Quadro 4) para o LVA3 e LV1, respectivamente, justamente os solos que apresentam os maiores teores de matéria orgânica (Quadro 1).

A capacidade máxima de adsorção de $Z n$ variou entre os sol os. A mais alta quantidade adsorvida foi de $2,18 \mathrm{mg} \mathrm{g}^{-1}$, encontrada no LV3, e a mais baixa foi de $1,11 \mathrm{mg} \mathrm{g}^{-1}$, encontrada no LV2 (Quadro 4). Para este el emento, o sol o mais argil oso (LV1) foi o que apresentou a maior afinidade de adsorção (Figura 1), o que mostra que, diferentemente do ocorrido com o $\mathrm{Cu}$, o teor de argila é mais determinante na adsorção de $\mathrm{Zn}$ que a matéria orgânica. Resultados semelhantes foram apresentados por Shuman (1975) e por Machado \& Pavan (1987), que também encontraram maiores valores de adsorção máxima de Zn para solos mais argilosos, independentemente dos teores de matéria orgânica.

O Cu apresentou maior energia de ligação aos sol os que o Zn (Quadro 4), graças, provavel mente, à mai or sel etividade das superfícies dos colói des para o $\mathrm{Cu}$, resultando em altas energias de ligação e estabilidade pela formação de complexos de esfera interna (Sparks, 1995). Este fato corrobora a menor disponi bi lização do el emento para as plantas nestes solos, em razão da baixa possibilidade de dessorção, como demonstrado por Nascimento (2001).

Comparando os valores de adsorção máxima de $\mathrm{Cu}$ e de Zn (Quadro 4) com os teores totais desses elementos nos solos (Quadro 1), percebe-se que muito pouco dos sítios disponíveis estão efetivamente ocupados por esses micronutrientes em condições 
Quadro 3. Isotermas de Langmuir (L) e Freundlich (F), em suas formas linearizadas, e coeficientes de correlação, ajustadas aos dados de adsorção de cobre e zinco em seis Latossolos

\begin{tabular}{|c|c|c|c|}
\hline Solo & Isoterma & Equação & $\mathbf{R}^{2}$ \\
\hline & \multicolumn{3}{|c|}{ Cobre } \\
\hline LV1 & $\mathrm{L}$ & $c / x / m=\quad 2,5348+0,5881 C$ & $0,98 * *$ \\
\hline & $\mathrm{F}$ & $\log x / m=-0,3569+0,333 \log C$ & $0,94 * *$ \\
\hline \multirow[t]{2}{*}{ LV2 } & $\mathrm{L}$ & $\mathrm{C} / \mathrm{x} / \mathrm{m}=16,695+0,9206 \mathrm{C}$ & $0,96 * *$ \\
\hline & $\mathrm{F}$ & $\log x / m=-0,8044+0,3945 \log C$ & $0,98 * *$ \\
\hline \multirow[t]{2}{*}{ LV3 } & $\mathrm{L}$ & $\mathrm{C} / \mathrm{x} / \mathrm{m}=13,6777+0,8469 \mathrm{C}$ & $0,96 * *$ \\
\hline & $\mathrm{F}$ & $\log x / m=-0,7440+0,3894 \log C$ & $0,98 * *$ \\
\hline \multirow[t]{2}{*}{ LVA1 } & L & $\mathrm{C} / \mathrm{x} / \mathrm{m}=28,821+0,9241 \mathrm{C}$ & $0,94 * *$ \\
\hline & $\mathrm{F}$ & $\log x / m=-1,0416+0,4875 \log C$ & $0,98 * *$ \\
\hline \multirow[t]{2}{*}{ LVA2 } & $\mathrm{L}$ & $\mathrm{C} / \mathrm{x} / \mathrm{m}=16,566+0,8392 \mathrm{C}$ & $0,94 * *$ \\
\hline & $\mathrm{F}$ & $\log x / m=-0,8235+0,4245 \log C$ & $0,98 * *$ \\
\hline \multirow[t]{3}{*}{ LVA3 } & $\mathrm{L}$ & $\mathrm{C} / \mathrm{x} / \mathrm{m}=3,475+0,4797 \mathrm{C}$ & $0,98 * *$ \\
\hline & $\mathrm{F}$ & $\log x / m=-0,4095+0,4067 \log C$ & $0,98 * *$ \\
\hline & \multicolumn{3}{|c|}{ Zinco } \\
\hline LV1 & $\mathrm{L}$ & $C / x / m=13,803+0,6198 C$ & $0,98 * *$ \\
\hline & $\mathrm{F}$ & $\log x / m=-0,8744+0,5326 \log C$ & $0,98 * *$ \\
\hline \multirow[t]{2}{*}{ LV2 } & $\mathrm{L}$ & $C / x / m=44,744+0,8963 C$ & $0,98 * *$ \\
\hline & $\mathrm{F}$ & $\log x / m=-1,3639+0,6352 \log C$ & $0,96 * *$ \\
\hline \multirow[t]{2}{*}{ LV3 } & $\mathrm{L}$ & $\mathrm{C} / \mathrm{x} / \mathrm{m}=59,378+0,4595 \mathrm{C}$ & $0,81 * *$ \\
\hline & $\mathrm{F}$ & $\log x / m=-1,6156+0,813 \log C$ & $0,98 * *$ \\
\hline \multirow[t]{2}{*}{ LVA1 } & L & $\mathrm{C} / \mathrm{x} / \mathrm{m}=81,247+0,7221 \mathrm{C}$ & $0,98 * *$ \\
\hline & $\mathrm{F}$ & $\log x / m=-1,7169+0,7776 \log C$ & $0,98 * *$ \\
\hline \multirow[t]{2}{*}{ LVA2 } & $\mathrm{L}$ & $C / x / m=80,988+0,5924 C$ & $0,90 * *$ \\
\hline & $\mathrm{F}$ & $\log x / m=-1,7500+0,8148 \log C$ & $0,98 * *$ \\
\hline \multirow[t]{2}{*}{ LVA3 } & $\mathrm{L}$ & $C / x / m=72,083+0,6773 C$ & $0,94 * *$ \\
\hline & $\mathrm{F}$ & $\log x / m=-1,6666+0,7744 \log C$ & $0,98 * *$ \\
\hline
\end{tabular}

**: Significativo a $1 \%$.
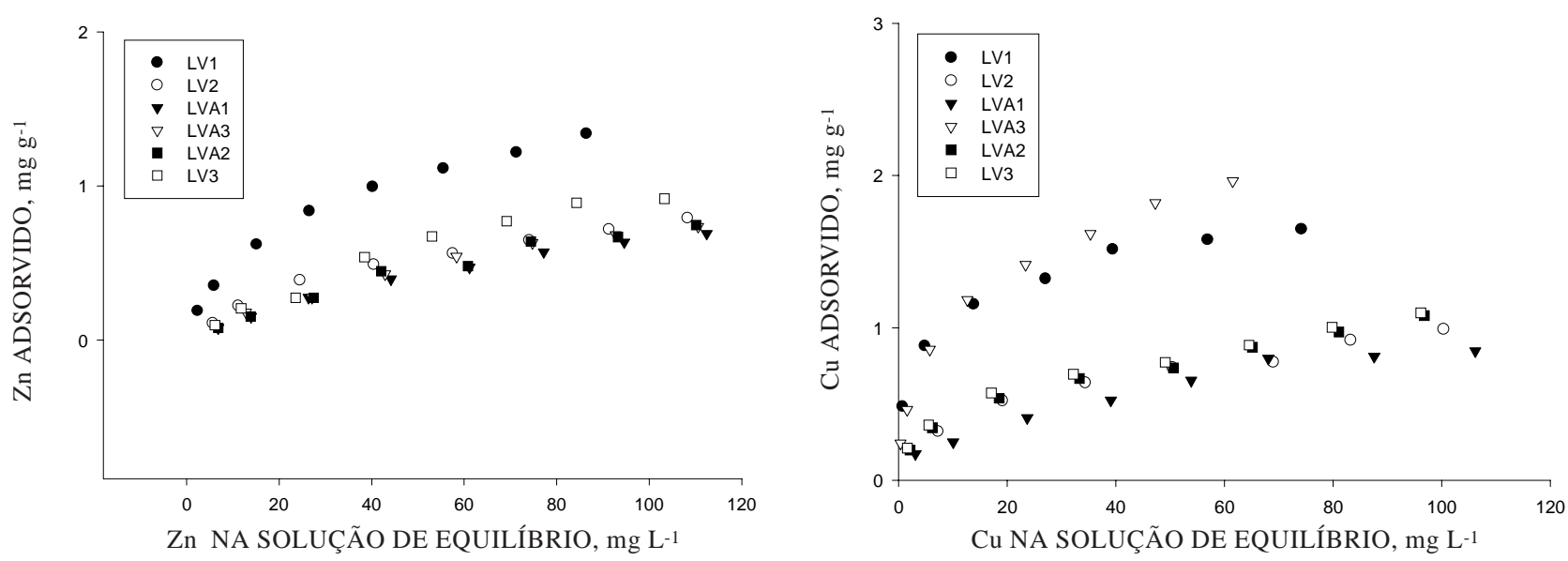

Figura 1. Relação entre os teores de zinco e de cobre adsorvidos na solução de equilíbrio em seis Latossolos.

naturais. Conseqüentemente, como também observado por McLaren \& Crawford (1973) para Cu, a força de retenção em baixa cobertura dos sítios de adsorção é, provavel mente, mais importante que a força de retenção média em toda a faixa de cobertura dos sítios, devendo ser determinante na disponibilidade desses elementos.
Para o Cu, a CTC efetiva e o teor de C orgânico apresentaram boas correlações com os parâmetros de adsorção da equação de Langmuir (energia de ligação e capacidade máxima de adsorção) e com a constante $k$ da equação de Freundlich (Quadro 5). Para o Zn, as melhores correlações com esses parâmetros foram obtidas com os teores de argila 
dos sol os, não havendo correlação significativa com os teores de C orgânico. Em concordância com Pulford (1986), Cunha et al. (1994) e Nascimento et al. (2002), as baixas correlações obtidas entre os parâmetros de adsorção de Zn com a CTC (Quadro 5) evidenciam a limitada importância da troca iônica na retenção de $Z n$ nos solos estudados. Em geral, as correlações obtidas confirmam os dados

Quadro 4. Parâmetros de ajuste das equações de Langmuir e $F$ reundlich para dados de adsorção de cobre e zinco em seis Latossolos

\begin{tabular}{|c|c|c|c|c|}
\hline \multirow{2}{*}{ Solo } & \multicolumn{2}{|l|}{ Langmuir } & \multicolumn{2}{|c|}{ Freundlich } \\
\hline & Energia de ligação & CMA & $\mathbf{K}$ & $\mathbf{n}$ \\
\hline & $\mathrm{L} \mathrm{mg}^{-1}$ & $\mathrm{mg} \mathrm{g}^{-1}$ & & \\
\hline \multicolumn{5}{|c|}{ Cobre } \\
\hline LV1 & 0,67 & 1,70 & 0,44 & 2,99 \\
\hline LV2 & 0,06 & 1,08 & 0,16 & 2,53 \\
\hline LV3 & 0,09 & 1,18 & 0,18 & 3,47 \\
\hline LVA1 & 0,04 & 1,08 & 0,09 & 2,05 \\
\hline LVA2 & 0,07 & 1,19 & 0,15 & 2,36 \\
\hline LVA3 & 0,60 & 2,08 & 0,39 & 2,46 \\
\hline \multicolumn{5}{|c|}{ Zinco } \\
\hline LV1 & 0,17 & 1,16 & 0,13 & 1,88 \\
\hline LV2 & 0,02 & 1,11 & 0,04 & 1,57 \\
\hline LV3 & 0,04 & 2,18 & 0,02 & 1,23 \\
\hline LVAI & 0,02 & 1,38 & 0,02 & 1,29 \\
\hline LVA2 & 0,02 & 1,69 & 0,02 & 1,22 \\
\hline LVA3 & 0,02 & 1,48 & 0,02 & 1,29 \\
\hline
\end{tabular}

Quadro 5. Correlações entre constantes das isotermas de Langmuir e Freundlich e características dos Latossolos

\begin{tabular}{|c|c|c|c|c|}
\hline \multirow{2}{*}{ Solo } & \multicolumn{2}{|l|}{ Langmuir } & \multicolumn{2}{|c|}{ Freundlich } \\
\hline & Energia de ligação & CMA & $\mathbf{K}$ & $\mathbf{n}$ \\
\hline \multicolumn{5}{|c|}{ Cobre } \\
\hline $\mathrm{pH}$ & $-0,38^{n s}$ & $-0,66^{* *}$ & $-0,41^{0}$ & $-0,08^{n s}$ \\
\hline Carbono & $0,69^{* *}$ & $0,90^{* *}$ & $0,65^{* *}$ & $-0,09 n s$ \\
\hline P-rem & $-0,32^{n s}$ & $-0,41^{0}$ & $-0,43^{0}$ & $-0,36^{n s}$ \\
\hline Argila & $0,09 s$ & $-0,17^{n s}$ & $0,15^{\text {ns }}$ & $0,24^{\text {ns }}$ \\
\hline CTCef & $0,98^{* *}$ & $0,96^{* *}$ & $0,96^{* *}$ & $0,15^{\text {ns }}$ \\
\hline \multicolumn{5}{|c|}{ Zinco } \\
\hline $\mathrm{pH}$ & 0,29 ns & $-0,17 \mathrm{~ns}$ & $0,33^{\text {ns }}$ & $0,37 \mathrm{~ns}$ \\
\hline Carbono & $-0,10^{n s}$ & $-0,08^{n s}$ & $-0,10^{\text {ns }}$ & $-0,12^{\text {ns }}$ \\
\hline P-rem & $-0,06^{n s}$ & $-0,12^{n s}$ & $-0,06^{\text {ns }}$ & $-0,08^{n s}$ \\
\hline Argila & $0,62^{* *}$ & $0,17 \mathrm{~ns}$ & $0,61^{* *}$ & $0,51^{*}$ \\
\hline CTCef & $0,55^{0}$ & $0,06^{n s}$ & $0,52^{*}$ & 0,39 ns \\
\hline
\end{tabular}

$\overline{n s, ~ 0,{ }^{*}} \mathrm{e}^{* *}$ : Não-Significativo e significativo a 10, 5 e $1 \%$, respectivamente. apresentados anteriormente, indicando, mais uma vez, que a matéria orgânica é o principal fator determinante na adsorção de Cu dos sol os estudados, enquanto, para o Zn, os teores de argila são mais importantes.

O P remanescente ( $\mathrm{P}$-rem), utilizado como medida correlacionada com a adsorção de $\mathrm{P}$ e de S e como estimativa da quantidade e qualidade da argila em solos (Alvarez V., 2000), apresentou baixa correlação com a adsorção máxima de Cu e não se correl acionou com os parâmetros de adsorção de Zn (Quadro 5).

Como sugerido por Couto et al. (1992), baseados no fato da al ta correlação entre o val or de $P$ rem e o coeficiente $\mathrm{Zn}$ recuperado/Zn adicionado em amostras de 12 solos, esperava-se que o $\mathrm{P}$ rem refletisse a adsorção de $\mathrm{Zn}$ nos solos, fato que não ocorreu. Deve-se destacar, no entanto, que Nascimento (2001) também encontrou correlação entre $\mathrm{P}$ rem e o coeficiente $\mathrm{Zn}$ recuperado/Zn para os mesmos sol os utilizados no presente trabal ho. É possível que essa discordância tenha ocorrido em virtude da utilização, neste experimento, de al guns solos com altos teores de matéria orgânica, notadamente os sol os LVA3 eLV1 (Quadro 1). Como já foi demonstrado, a adsorção de Zn está relacionada com os teores deargila enão com os teores dematéria orgânica (Quadro 5). O baixo valor de P na solução de equilíbrio para estes solos parece, entretanto, ter sido decorrente, principalmente, da sorção pela matéria orgânica, visto que o menor val or de $P$ rem foi obtido para o LVA3 (Quadro 1), solo com maior teor de C orgânico. O resultado indica, portanto, que as diferenças em teores de matéria orgânica entre os solos e a maior afinidade entre $\mathrm{Zn}$ e superfícies dos minerais relativamente aos complexos orgânicos podem ser responsáveis pela ineficiência do $\mathrm{P}$ rem em refletir adequadamente a capacidade de adsorção de $\mathrm{Zn}$ nestes solos.

\section{CONCLUSÕES}

1. As equações de Langmuir e F reundlich foram eficientes na determinação dos parâmetros de adsorção de Zn e Cu nos solos, sendo os teores de argila e de matéria orgânica, respectivamente, as características mais bem relacionadas com a capaci dade de adsorção desses el ementos pel os sol os.

2. Os solos apresentaram maior energia de ligação dos sítios de troca e maior capacidade de adsor ção máxima para Cu relativamente ao Zn.

3. O P remanescente não se correlacionou com os parâmetros de adsorção de Zn, em virtude, provavelmente, da influência da matéria orgânica em sua determinação. Este fato pode limitar sua eficiência na previsão da adsorção deste elemento em sol os com teor alto de carbono orgânico. 


\section{LITE RATURA CITADA}

ALVAREZ V., V.H.; NOVAIS, R.F.; DIAS, L.E. \& OLIVEIRA, J .A. Determinação e uso do fósforo remanescente. Viçosa, Sociedade Brasileira de Ciência doSolo, 2000. 32p. (Boletim informativo, 25)

BIBAK, A. Competitive sorption of copper, nickel, and zinc by an Oxisol. Comm. Soil Sci. Plant Anal., 28:927-937, 1997.

COUTO, C.; NOVAIS, R.F.; TEIXEIRA, J .L; BARROS, N.F. \& NEVES, J .C.L. Níveis críticos de zinco no solo e na planta para o crescimento de milho em amostras de solos com diferentes valores do fator capacidade. R. Bras. Ci. Solo, 16:79-87, 1992.

CUNHA, R.C.A.; CAMARGO, O.A. \& KINJ O, T. Aplicação de três isotermas na adsorção de zinco em Oxissolos, Alfissol os e Ultissolos. R. Bras. Ci. Solo, 18:15-20, 1994.

De FILIPPO, B.V. \& RIBEIRO, A.C. Análise química do solo metodologia. 2.ed. Viçosa, U niversidade F ederal de Viçosa, 1997. 26p.

ECHEVERRÍA, J.C.; MORERA, M.T.; MAZKIARÁN, C. \& GARRIDO, J J . Competitive sorption of heavy metal by soils. Isotherms and fractional factorial experiments. Environ. Poll., 101:275-284, 1998.

EMPRESA BRASILEIRA DE PESQUISA AGROPECUÁRIA EMBRAPA. Manual de métodos de análise de solo. 2.ed. Rio deJ aneiro: Centro Nacional dePesquisa de Solos, 1997. 212p.

HARTER, R.D. Micronutrient adsorption-desorption reactions in soils. In: MORTVERDT, J .J .; COX, F.R.; SHUMAN, L.M. $\&$ WELCH, R.M., eds. Micronutrients in the agriculture, Madison, Soil Science Society of America, 1991. p.59-88.

LOPES, A.S. \& ABREU, C.A. Micronutrientes na agricultura brasileira: evolução histórica e futura. In: NOVAIS, R.F.; ALVAREZ V., V.H. \& SCHAEFER, C.E.G.R., eds. Tópicos em Ciência do Solo, Viçosa, Sociedade Brasileira de Ciência do Solo, 2000. v.1. p.265-298.

MACHADO, P.L.O.A. \& PAVAN, M.A. Adsorção de zinco por alguns sol os do Paraná. R. Bras. Ci. Solo, 11:253-256, 1987

MARTÍNEZ, C.E. \& MOTTO, H.L. Solubility of lead, zinc and copper added to mineral soils. Environ. Poll., 107:153-158, 2000.

McBRIDE, M.B. \& BLASIAK, J J . Zinc and copper solubility as a function of pH in an acid soil. Soil Sci. Soc. Am. J ., 43:866870, 1979.

MCLAREN, R.G. \& CRAWFORD, D.V. Studies of soil copper. II. Thespecific adsorption of copper by soils. J . Soil Sci., 24:445452, 1973.
MESQUITA, M.E. Copper and zinc competitive adsorption in schistic and granitic acid soils. Agrochimica, 17:235-244, 1998.

MSAKY, J J . \& CALVET, R. Adsorption behavior of copper and zinc in soils: influence of $\mathrm{pH}$ on adsorption characteristics. Soil Sci., 150:513-522, 1990.

NASCIMENTO, C.W.A. Dessorção, extração e fracionamento de zinco, cobre e manganês em solos. Viçosa, U niversidade Federal de Viçosa, 2001. 60p. (Tese de Doutorado)

NASCIMENTO, C.W.A.; FONTES, R.L.F.; NEVES, J .C.L. \& MELÍCIO, A.C.F.C. Fracionamento, dessorção e extração química de zinco em Latossolos. R. Bras. Ci. Solo, 26:599606, 2002.

PETRUZZE LI ET, G.; GUIDI , G. \& LUBRANO, L. I onic strenght effect on heavy metal adsorption by soil. Comm. Soil Sci. Plant Anal., 16:971-986, 1985.

POMBO, L.C.A. \& KLAMT, E. Adsorção de zinco e cobre de dois solos do estado do Rio Grande do Sul. R. Bras. Ci. Solo, 10:191-194, 1986.

PULFORD, I.D. Mechanisms controlling zinc solubility in soils. J. Soil Sci., 33:427-438, 1986.

RESENDE , L.C.A.M.; BAHIA FILHO, A.F.C. \& BRAGA, J .M. Mineralogia de Latossolos estimada por alocação a partir do teor total de óxidos do ataque sulfúrico. R. Bras. Ci. Solo, 11:1-23, 1987.

SELIM, H.M. Modelling thetransport and retention of inorganics in soils. Adv. Agron., 47:331, 1992.

SHUMAN, L.M. The effect of soil properties on zinc adsorption by soils. Soil Sci. Soc. Am. Proc., 39:454-458, 1975.

SILVEIRA, M.L.A. \& ALLEONI, L.R.F. Copper adsorption in tropical soils. Braz. Arch. Bio. Tech., 46:529-536, 2003.

SILVEIRA, M.L.A.; ALLEONI, L.R.F.; CAMARGO, O.A. \& CASAGRANDE, J .C. Copper adsorption in oxidic soils after removal of organic matter and iron oxides. Comm. Soil Sci. Plant Anal., 33:3581-3592, 2002.

SPARKS, D.L. Sorption phenomena on soils. In: SPARKS, D.L., ed. Environmental soil chemistry. San Diego, 1995. p.99139.

URE, A.M. Methods of analysis for heavy metals in soils. In: ALLOWAY, B.J ., ed. Heavy metals in soils. New York, J ohn Wiley \& Sons, 1990. p.40-80.

VETTORI, L. Métodos de análise de solo. Rio de J aneiro, Ministério da Agricultura, Equipe de Pedologia e Fertilidade do Solo, 1969. 24p. (Boletim técnico, 7)

YUAN, G. \& LAVKULICH, L.M. Sorption behavior of copper, zinc, and cadmium in response to simulated changes in soils properties. Comm. Soil Sci. Plant. Anal., 28:571-587, 1997. 